\title{
Healthcare Logistics Optimization Framework for Efficient Supply Chain Management in Niger Delta Region of Nigeria
}

\author{
Imeh J. Umoren ${ }^{1}$, Ubong E. Etuk ${ }^{2}$, Anietie P. Ekong ${ }^{3}$, Kingsley C. Udonyah ${ }^{4}$ \\ Department of Computer Science, Akwa Ibom State University \\ Mkpat Enin, Akwa Ibom State, Nigeria
}

\begin{abstract}
Optimizing logistics allocation and utilization is essential for effective healthcare management. Apparently, less consideration is given to it in most hospitals in Nigeria where less resources are allocated to health sector in yearly budgetary. Hospital consists of several patient classes, each of which follows different treatment process flow paths over a multiphase and multidimensional requirement with scarce resources and inadequate space. Despite the small budget provision made for healthcare resources, patient's demands for better service is rapidly experiencing upsurge. Hence, efficient and optimal solutions are required to lessen costs of healthcare service towards enhancing Quality of Care (QoC) and Quality of Experience (QoE) in most public healthcare sector. However, certain control coefficients like the absence of a Dedicated Logistics Department (DLD) in the medical facilities actually limit the efforts of stakeholders. This paper proposed a Computational framework to assess various strategic and operational decisions for optimizing the multiple objectives using Type-1 Fuzzy Logic Model. In phase I, we explore healthcare resource allocation plan. In phase II, we determine a resource utilization schedule by patient class for daily operational level. While in Phase III, we develop a framework capable of evaluating and optimizing healthcare logistics using control coefficients of Logistics Optimization (LO), Integration of Information/Cognitive Technologies (ETA), and Collaboration of all Logistics Stakeholders (COL). We assigned weights between 1 and 10 to the coefficients and modeled the effects on efficient supply chain. Finally, we further explore the effects of separate strategies and their combination to identify the best possible resource supply chain. The computational experiment was considered on the basis of data obtained from a study conducted on a typical public healthcare department. Results show that our approach significantly evaluate and optimized healthcare logistics.
\end{abstract}

Keyword-Dedicated logistics department (DLD); Quality of Care (QoC); Quality of Experience (QoE); information/cognitive technologies (ETA) and type-1 fuzzy logic model

\section{INTRODUCTION}

The current state of Healthcare Service Delivery (HSD) in the South-South region of Nigeria - and in many other regions of the country is very precarious.

There are numerous cases where both healthcare providers or managers and healthcare users going through critical deprivation - on one hand, healthcare managers are unable to do their jobs while on the other hand, the poor masses requiring healthcare services and other healthcare users suffer health challenges and even death. This critical challenge that have been recorded in this region can be traceable to unavailability of very required medical consumables, including medical professionals - like nurses and doctors, often resulting from many factors, one of which is lack of logistic framework for Supply Chain in the south-south, Nigeria, resulting in many deaths recorded in the emergency units of the hospitals. In one instance, University of Uyo Teaching Hospital (UUTH), many Psychiatric patients that required hospitalization were not hospitalized due to inadequate bed spaces as most hospital in Nigeria only has 0.4 psychiatric beds per 1000 population [25] Despite the fact that the South-South region of the country accounts for a great majority of the primary natural resources (crude oil) as the country exports, the region is highly neglected by the government in the provision of healthcare services. The government owned healthcare centers in the region are in many cases ill-equipped or none existent or is neglected altogether by the relevant stakeholders while most of the functioning healthcare centres are very short of personnel. In many cases, only one qualified nurse or doctor may be assigned to the centre and thus cannot handle the demands of providing quality healthcare services to the ever-increasing populace. Furthermore, due to the deplorable state of the healthcare centres,

Similarly, private healthcare centers owned by individuals are very expensive. Hence, cannot be afforded by the masses leaving in these regions who are mostly petty traders, farmers, fishermen, civil servants and the likes. The effect arising from the fact that there are no logistics evaluation framework put in place by healthcare stakeholders to address this situation. The private owned healthcare centers though functioning and equipped are still unable to meet the demands placed on them. There are still cases of running out of medical consumables because the required framework is not put in place to manage the logistics that will ensure an efficient supply chain. [7], portrays a very damning reality: Indeed, healthcare cost are increasing and healthcare managers are under increasing pressure to reduce the cost of healthcare provision - with patients expecting high quality care at affordable cost at reduced cost: there is need for better logistics practices.

A key characteristic of this region in terms of its geographical nature, is that it is swampy and thus provides enough breeding ponds for mosquitoes to thrive - as a result 
the outbreak of malaria is high and the deaths recorded from malaria in this region is higher than those obtained elsewhere within the country. of the six states that constitutes SouthSouth: Akwa Ibom, Bayelsa, Cross River, Delta, Edo, and Rivers, only Akwa Ibom State have improved impressively in sponsoring and ensuring healthcare service delivery due to the investment of the Akwa Ibom state government within the past eight years thus providing free medical care to pregnant women, children and nursing mothers and this was made possible because of proper logistics implementation. Evidently, logistics activities in healthcare centres, hospitals or clinics have been indicated to provide a significant avenue for cost containment in healthcare if best practices are implemented [7] Since businesses including healthcare industries depend on their Supply Chains to provide them with what they need to survive and thrive [11] there is validation to develop a framework that could ensure a more efficient supply chain.

\section{RELATED WORKS}

Not much research works has discussed the concept of healthcare logistics evaluation for the purpose of creating a more efficient supply chain for the healthcare organization. Nevertheless, many scholars have handled to a very great extent very important subjects that constitute the core of our discuss in this work. In the following paragraphs we present a summary of related works that lends credence to the subject of healthcare logistics evaluation framework for efficient supply chain. [13] established that Hospital logistics, viewed as a vital part of a hospital that is in charge of purchasing, receiving, stock management etc., accounts for up to $46 \%$ of hospital budget - and since this amount is considered a very substantial proportion, especially in the context of budgetary restrictions applied to all organizations including hospitals and healthcare centers, they identified exhaustively logistics activities based on determining logistics manifestations within hospitals-healthcare centers. They also performed the organization and management of these activities in order to point out the departments or services, in the healthcare institutions, that handles them-this they achieved through a comparison between the various countries. They adopted a literature-review based methodology where they reviewed over 60 papers published between 2000 and 2017. From the various hospitals studied in selected countries includingFrance, Quebec, United States of America and Morocco, they presented the following departments and the activities they controlled or discovered as shown in Table I.

From the study summarized in Table I, we see that a singular logistics activity is handled by more than one department - this is known to create delays in the logistics process and increase the bottleneck existing in hospital logistics. Additionally, from this we discover, the diversity of logistics activities; where different departments are involved in the management of logistics activities and where some of the logistics activities are outsourced. The Administrative Department is obviously involved in too many of the activities resulting serious overhead and increased workload-delaying the time constraint of the logistics process. Noticeably, the table above showed that no Dedicated Department has been created solely for the purpose of managing healthcare logistics - a move that would have ensured efficient supply chain management!

TABLE I. Logistics Activities AND DEPARTMENTS IN CHARge (Source: [13])

\begin{tabular}{|c|c|c|c|c|c|c|}
\hline Logistics Activities & $\begin{array}{l}\text { Medical } \\
\text { Affairs } \\
\text { Division }\end{array}$ & $\begin{array}{l}\text { Nursing Care } \\
\text { Division }\end{array}$ & $\begin{array}{l}\text { Administrative } \\
\text { Affairs Division }\end{array}$ & $\begin{array}{l}\text { Medical } \\
\text { Departments }\end{array}$ & $\begin{array}{l}\text { Pharmacy } \\
\text { Service }\end{array}$ & $\begin{array}{l}\text { Reception and } \\
\text { Admission Service }\end{array}$ \\
\hline Scheduling & $\checkmark$ & $\checkmark$ & & $\checkmark$ & & \\
\hline Procurement & & & $\checkmark$ & & & \\
\hline Distribution & & & $\checkmark$ & & & \\
\hline Pharmacy & $\checkmark$ & & $\checkmark$ & & $\checkmark$ & \\
\hline Catering/ Food & & & $\checkmark$ & & & \\
\hline Laundry & & & $\checkmark$ & & & \\
\hline Hygiene & & & $\checkmark$ & & & \\
\hline Waste Management & & & $\checkmark$ & & & \\
\hline Maintenance & & & $\checkmark$ & & & \\
\hline Reception Service & & & & & & $\checkmark$ \\
\hline Patient Flow & & & & & & $\checkmark$ \\
\hline Telecommunication & & & $\checkmark$ & & & \\
\hline $\begin{array}{l}\text { Information System } \\
\text { Management }\end{array}$ & & & & & & $\checkmark$ \\
\hline Stock Management & & & $\checkmark$ & & & \\
\hline Mail Service/ Files Archiving & $\checkmark$ & & $\checkmark$ & & & $\checkmark$ \\
\hline Safety and Security & & & $\checkmark$ & & & \\
\hline
\end{tabular}


In [19], in his study on the assessment of logistics management - a study carried out in Chana Health services, ran a 3-tier system of managing medical consumables (health commodities), suppliers, the central medical store, the regional medical store, service delivery points and the transportation system. These they illustrate to form the supply chain. They adopted a multi-case study approach to assess the practices of logistics management and with these they discovered the causes of inadequacy of logistics in Ghana as well as the strengths and weaknesses of the Ghanaian Health Services' System. Many scholars have proposed numerous approaches for curbing the challenges associated with logistics management and supply chain integration. In one of these studies on Structured Review of Quantitative Models of the Pharmaceutical Supply [3] identified and provided a structured overview of quantitative models in the pharmaceutical supply chain as a means for optimizing the supply chain process. The author in [10] suggested door to door service as a means for ensuring effective healthcare supply chain based on their work on scheduling Optimization of Home Healthcare Services. They proposed that the reasonable arrangements of nurses and their routes will not only reduce medical expenses but can also enhance patient's satisfaction. In further studies, [9] after analyzing the literatures relating to the dimensions of healthcare logistics and supply chain management, shows that the areas of hospitals interfere with the determinants of satisfaction as well as the care quality standards. Inferring from the above, it could be concluded that improving the quality-of-care services and hence efficient supply chain is dependent on the efficiency of the logistics activities within the healthcare institution that will enhance efficient management. The author in [9] adopted a methodology based on the synthesis of articles and scientific reports dealing with key words/phrases of the subject matter including quality of care, hospital logistics etc. They gathered that to achieve high and improved quality, healthcare of institutions may adopt a variety of approaches, one of which is the optimization of their logistic - and this have been proposed by several scholars as one of the most efficient ways to improve both the quality of care and a more efficient management of the Healthcare institutions [16]; [9]., establishes a link between hospital logistics and quality of care and examines how strong the impact of an effective logistics is on the delivery of high quality. A Simulation-Based Multi Objective Optimization approach for Healthcare Services Management, a novel approach was proposed for healthcare services management [20]; [10]. Specifically, the use of a simulation optimization approach was prescribed for the optimal resources allocation to wards in very big hospitals. The proposed simulation-based optimization approach is based on a discrete event simulation model reproducing the hospital services and combined with a derivative-free multi objective optimization method. The results obtained on the obstetrics ward of an Italian hospital are reported, showing the effectiveness of the new approach proposed. From the methodological point of view, the main contribution of this work is the use of a simulation optimization framework, which integrates a DES model and an optimization algorithm, allowing studying the problem in hand as a multi objective Optimization problem. Then, the DFMO algorithm used enables to obtain an approximate Pareto set of points.

\section{LOGISTICS VARIABLES FOR EFFICIENT SUPPLy CHAIN}

Several works have discussed key logistics variables [7]; [13] to consider for achieving specified improvements in the healthcare institutions including less cost of healthcare service; a more efficient service delivery and efficient supply chain. A cross-section of some key logistics key variables or factors are presented in Table II.

The author in [23], examined how modularity is used for enabling value creation in managing healthcare logistics services. They applied materials logistics of four different kinds of hospitals was examined through a qualitative case study. They built a theoretical framework on literature on healthcare logistics, service modularity and value creation. From the findings, their case hospitals were discovered to have developed their material logistics independently from others when looking at the modularity of offerings, processes and organizations. Services such as assortment management, shelving and developing an information platform, have been performed in-house partly by the care personnel - although should be managed by a Dedicated Logistics Department (DLD). However, steps toward modularized and standardized solutions are now being taken in the case hospitals, including ideas about outsourcing some services. Many scholars agree on centralization as being the best approach to effect an efficient supply chain but [23] maintains that modularity offers a tool for developing logistics services inside the hospitals and increases possibilities to consider also external logistics service providers.

The author in [26] postulated that, the diagnoses of diseases are carried out by medical experts with professional experience and adequate knowledge capable of identifying diseases on clinical data of patients, but such diagnosis is found to be approximate and time-consuming. The paper proposed an enhanced approach to be considered to mitigate the time-consuming nature of disease diagnoses.

Developing a framework for evaluating healthcare logistics is a conceptual equivalence of building an actual Healthcare Management System (HMS). Hence, the qualities considered when developing a system should also be considered when developing an evaluation framework. The author in [19] presented a list of quality attributes that should be considered in creating both a framework and system. This is presented in Table III.

Logistics Optimization has been earmarked as a key driver for achieving a sustainable supply chain [12]; [6] and having a centralized warehouse has been proposed as an ideal choice for healthcare institutions. The purpose for designing a framework for the evaluation of healthcare logistics is to achieve an efficient supply chain supply chain in the southsouth of Nigeria and this is achieved through logistics evaluation and optimization. 
TABLE II. KEY LOGISTICS VARIABLES

\begin{tabular}{|c|c|c|}
\hline Variables & Applicability & Authors \\
\hline Strategic Sourcing & \multirow{3}{*}{$\begin{array}{l}\text { Logistics and Supply Chain Strategizing } \\
\text { (Strategy) }\end{array}$} & \multirow{7}{*}{ [27] } \\
\hline Supply Chain Network Design & & \\
\hline Product design \& development & & \\
\hline Demand Planning & \multirow{4}{*}{$\begin{array}{l}\text { Logistics and Supply Chain Operations } \\
\text { (Operations) }\end{array}$} & \\
\hline Procurement & & \\
\hline Inventory & & \\
\hline Logistics & & \\
\hline Quality of Services & \multirow{3}{*}{$\begin{array}{l}\text { Logistics and Supply Chain Evaluation } \\
\text { (Evaluation) }\end{array}$} & \multirow{7}{*}[7]{} \\
\hline Process Complexity & & \\
\hline Staff Competence/Skills & & \\
\hline Inventory Management & \multirow{4}{*}{$\begin{array}{l}\text { Logistics and Supply Chain Implementation } \\
\text { (Implementation) }\end{array}$} & \\
\hline Process Efficiency & & \\
\hline Supply Chain (SC) Integration & & \\
\hline Cost of Implementing & & \\
\hline
\end{tabular}

TABLE III. CATEGORIES/SUb-CATEGORIES OF FRAMEWORK QUALITY ATtRIBUtES

\begin{tabular}{|l|l|l|}
\hline SN & Categories & Sub-Categories \\
\hline 1. & Functional Suitability & Functional completeness, functional correctness, functional correctness, \\
\hline 2. & Performance Efficiency & Time behaviour, Resource Utilization, Capacity \\
\hline 3. & Compatibility & Co-existence, interoperability \\
\hline 4. & Usability & Appropriateness, recognisability, Learnability, Operability, User error protection, Accessibility \\
\hline 5. & Reliability & Maturity, Availability, Fault Tolerance, Recoverability \\
\hline 6. & Security & Confidentiality, Integrity, Non-repudiation, Accountability, Authenticity \\
\hline 7. & Maintainability & Modularity, Reusability, Analyzability, Modifiability, Testability, Portability, Adaptability, Installability, Replaceability \\
\hline 8. & Effectiveness & Value ability, Specificity, Adequately perform intended task. \\
\hline 9. & Efficiency & Solve intended tasks with minimal resource usage \\
\hline 10. & Satisfaction & Usefulness, Trust, Pleasure, Comfort \\
\hline 11. & Minimal Risk & Health \& Safety Risk mitigation, Economic \& Environmental Risk mitigation \\
\hline 12. & Context Coverage & Context completeness, flexibility \\
\hline
\end{tabular}

\section{THE COMPUTATIONAL FRAMEWORK}

We present a comprehensive and in-depth study of existing frameworks within the field of healthcare Logistics and Supply Chain Management in order to identify the weaknesses and functional limitations of the system. This paper employed this approach in a bid to garner a clear insight and understanding the dynamics, functionalities and requirements as well as existing knowledge as presented by reputable scholars whose works we have reviewed, required for developing an evaluation framework. With a properly structured analyses, the questions and issues to be addressed, the strengths and weaknesses, characteristics and requirements of the existing frameworks have been identified. The sole objective of carrying out this analysis, within the scope of the subject area, is to pinpoint the quality attributes, logistics variables/drivers, supply chain drivers as well as the mode of operation of the existing frameworks and their limitations with the view of evaluating and optimizing, and solving the problems and bottlenecks associated with healthcare logistics and supply in the south-south of Nigeria.

This section considers the methods and methodologies applied to logistics and supply chain management proposed and used by scholars whose work we have reviewed in the previous chapter. The methodology used in this research work is adequately explained. It outlines the skeletal approach and design of the proposed framework.

\section{A. Logistics Evaluation/Optimization Framework}

The past three decades have witnessed the proposition and adaptation of several problem-specify methodologies. However, before the emergence of these specified methodologies and algorithm for solving problems - that could be applied repeated to get the same results at different places and in different times, randomized controlled trails 
were the most reliable method of determining effectiveness [2]. However, these randomized controlled trials were discovered to have very high cost and time constraints. Often, it would take several days or several weeks to give a solution using this methodology as several randomized trials have to be made and then the most efficient trial approach that produced the best solution is chosen. This is not always the case with these trials. In many cases, no optimal solution is found even after several weeks and this validates the need for a better framework.

The introduction of a dedicated logistics department will immediately eliminate the need for retailers thus reducing the associated costs and speeding up the process and data flow. The dedicated logistics department could hitherto interact directly with the plants (producers/manufacturers) depending on the capacity of the healthcare institution or costs constraints involved with interacting with the plants and they can also interact with the suppliers thus cutting off costs associated with interacting with middlemen or the retailers. In Fig. 1, the Dedicated Logistics Department is saddled with making the decision of which is the optimal procurement option based both on transportation costs, product quality and the time between request and delivery.

\section{B. Supply Chain Structure/Configuration}

Supply Chain has been integrated into the healthcare organization as a new way approach to conceptualizing medical procurement, inventory and supply management [14]. Healthcare Supply Chain is seen by [14] as the information, supplies and finances involved with the acquisition and movement of goods and services from the supplier to the end user in order to enhance clinical outcomes while controlling costs. From this, we see that the key focus is to enhance clinical outcomes (better services) and controlling or reducing cost - which should be ensured with implementing an efficient supply chain through evaluation of Healthcare Logistics. The supply chain structure is a complex one to define when considered in a whole with all the components that it incorporates however, the structure is quite simple and easy to graphically represent when conceptualized in smaller abstractions. We present a simple abstraction of the Supply Chain Structures that contribute to form the complete supply chain structure.

The simple supply chain abstraction/configuration considers three very important drivers: Suppliers, Company and Customers or end users. Our focus here is the companythe healthcare institution. Once abstraction of this driver is expanded, it embodies a whole lot of other drivers which are discussed in the subsections that follow. From our study, we have identified that the supply chain structure used in the several of the healthcare centres in the Niger Delta region of Nigeria is the very Extended Supply Chain Structure, with so many players involved between the producing firms and the final costumer or ultimate consumer. This is responsible for over $78 \%$ of the costs and time constraints involved in the supply chain structure used in the Niger Delta. Fig. 2 indicates a Simplified (Optimized) Supply Chain Structure.

\section{Evaluation Framework Design Considerations}

Based, majorly, on our literature review and the data gotten from the observation of the Medical facilities selected for our case study, we present a Healthcare Logistics Evaluation Framework (HLEF) for and propose the enactment of a Dedicated Logistic Department (DLD) in all healthcare facilities for Efficient Supply Chain Management (ESCM) in all Healthcare Institutions. The methodologies for the design and operation of the Supply Chain Network or Logistics Evaluation Framework is either steady state, dynamic (for which a computational intelligence approach is employed), deterministic or such that it could deal with uncertainties associated with demands for healthcare service or medical consumables. Considering that we are integrating Continuous Improvement $(\mathrm{CI})$ as a design consideration for our proposed framework, a dynamic model capable of dealing with the uncertainties that may arise in future within the healthcare landscape has been adopted using Fuzzy Logic.

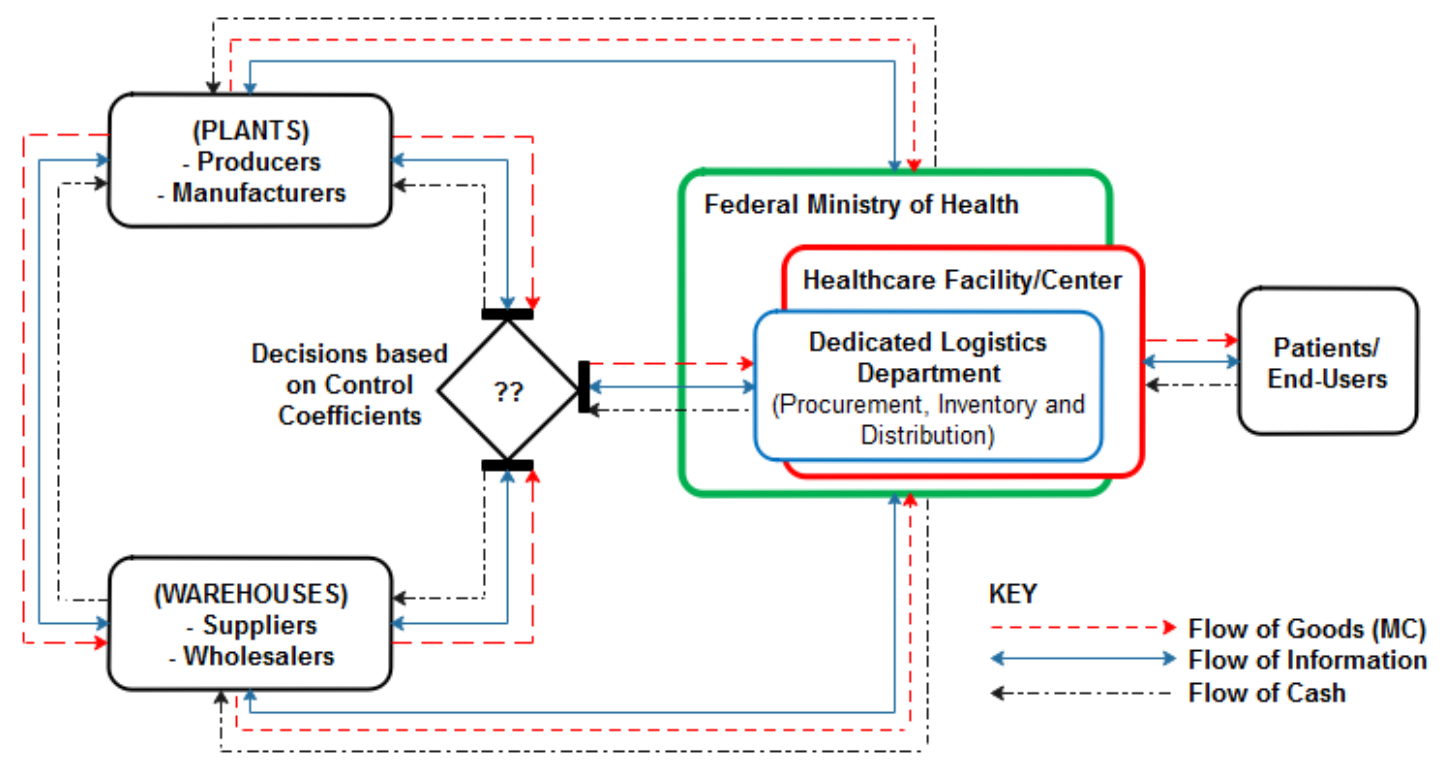

Fig. 1. Our Enhanced Healthcare Logistics Network Configuration. 


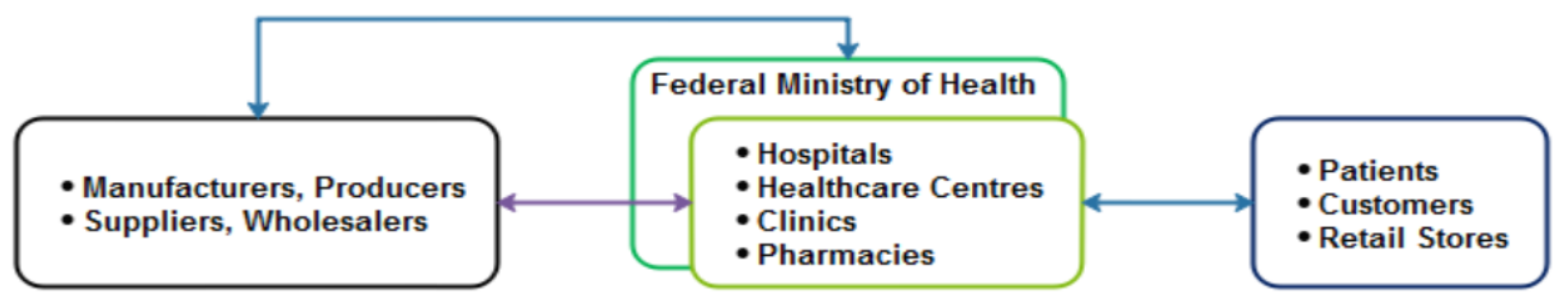

Fig. 2. A Simplified (Optimized) Supply Chain Structure (Adapted [11]).

\section{Fuzzy based Framework}

To development a robust Healthcare Logistics Evaluation and Optimization framework for efficient Supply Chain, we employ the combination of a multi-criteria and computational intelligence approach using Fuzzy Logic Type-1 to design a framework that implements a Dedicated Logistics Department (DLD). The Multiple criteria Technique presents modalities for implementing efficient supply chain based chosen key logistics variables (as presented in Table IV). Importantly, the absence of a Dedicated Logistics Department (DLD) and Lack of Logistics Optimization Strategy has been identified as the major control coefficients with the Highest Impact factors, using the Six Sigma framework, on the Efficiency Index of the Healthcare Supply Chain. Combining these two robust techniques, we present intelligent evaluation and optimization framework for healthcare logistics targeted at realizing an efficient supply chain. Based on this premise, we defined our fuzzy rules based on the following if-then - else Fuzzy rule implementation algorithm.
1) 3.41 Fuzzy rules definition: To implement the optimization module of our evaluation and optimization framework, we have defined the rules following laid down fuzzy rules. The form of a fuzzy rule is defined as a conditional statement. The fuzzy rules are defined using the standard;

$R^{1}: I F x_{1}$ is $F_{1}^{i}$ and $\ldots x_{p}$ is $F_{p}^{l}$ THEN $y$ is $G_{1}^{l}$

Where $1=1, \ldots ., \mathrm{M}$

From our structured analysis, we have identified (4) input variables and each of these variables have three (3) sets each. From combinational logic, we understand that a truth table of $N$ inputs contains $2^{N}$ rows, one for each possible value of the inputs. From the 4 input variables, the maximum possible number of rules to be used in defining our rule base is given as $3^{4}=81$. As stated earlier, the rule is a collection of $I F-$ THEN statements. The Linguistic Variables are shown in Table IV and the $I F-T H E N$ rules are shown in the Table V.

TABLE IV. LINGUISTIC VARIABLES

\begin{tabular}{|l|l|l|}
\hline SN & Linguistics Variables & Key \\
\hline 1. & Logistics Optimization & LO \\
\hline 2. & Information/Cognitive Technology Adaptability & ETA \\
\hline 3. & Strategic collaboration of all Stakeholders and Suppliers & COL \\
\hline 4. & Implementation of Dedicated Logistic Department & DLD \\
\hline
\end{tabular}

$$
\begin{gathered}
\text { IF - THEN RULES } \\
\text { If }
\end{gathered}
$$

All four parameters are at their highest then SCE is Efficient

Else if

DLD is adopted and at least any two other key variable is at their highest

\section{Then SCE is Efficient}

\section{Else if}

DLD is adopted and any other key variable is at its highest value

Then SCE is average

Else if

any three key variables are at its highest value

Then SCE is average

Else

SCE is Not Efficient 
TABLE V. RULE BASE

\begin{tabular}{|c|c|c|c|c|c|}
\hline Rule No. & LO & ETA & COL & DLD & SCE \\
\hline & Poor & None & High & None & Inefficient \\
\hline & Poor & None & Medium & None & Inefficient \\
\hline & Poor & None & Low & None & Inefficient \\
\hline & Poor & Minimal & High & None & Inefficient \\
\hline & Poor & Minimal & Medium & None & Inefficient \\
\hline & Poor & Minimal & Low & None & Inefficient \\
\hline & Poor & Maximal & High & None & Inefficient \\
\hline & Poor & Maximal & Medium & None & Inefficient \\
\hline & Poor & Maximal & Low & None & Inefficient \\
\hline & Good & None & High & None & Average \\
\hline & Good & None & Medium & None & Inefficient \\
\hline & Good & None & Low & None & Inefficient \\
\hline & Good & Minimal & High & None & Average \\
\hline & Good & Minimal & Medium & None & Average \\
\hline & Good & Minimal & Low & None & Inefficient \\
\hline & Good & Maximal & High & None & Average \\
\hline & Good & Maximal & Medium & None & Average \\
\hline & Good & Maximal & Low & None & Inefficient \\
\hline & Excellent & None & High & None & Inefficient \\
\hline & Excellent & None & Medium & None & Inefficient \\
\hline & Excellent & None & Low & None & Inefficient \\
\hline & Excellent & Minimal & High & None & Average \\
\hline & Excellent & Minimal & Medium & None & Average \\
\hline & Excellent & Minimal & Low & None & Inefficient \\
\hline & Excellent & Maximal & High & None & Average \\
\hline & Excellent & Maximal & Medium & None & Average \\
\hline & Excellent & Maximal & Low & None & Inefficient \\
\hline
\end{tabular}

To implement the rules in Table V, we used the MATLAB Type-1 Fuzzy Logic Toolbox to realize the steps needed to actualize this framework implementation, shown in Fig. 3.

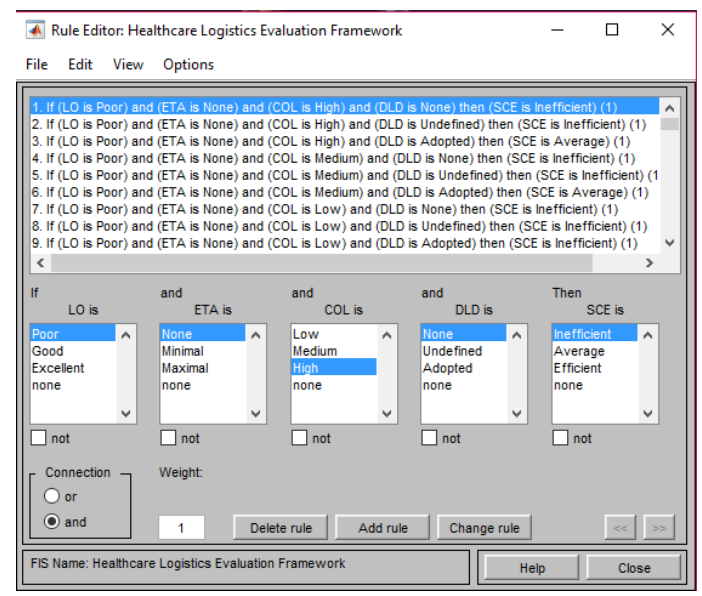

Fig. 3. Fuzzy Rule Editor.

\section{E. Fuzzy Logic System}

The conceptual system architecture used for this work is based on the Fuzzy Inference System that basically consists of the MATLAB Graphical User Interface that provides the platform for defining the Fuzzy Rules and Membership Function. The conceptual architecture illustrated in Fig. 4 consists of the Knowledge Engine, Knowledge Base which is made of the database model and the Fuzzy Logic Model and the user Interface. The knowledge engine consists of structured and unstructured data, but in this work structured data (Logistics Optimization (LO), Information/Cognitive Technology Adaptation (ETA), Strategic Collaboration of all Stakeholders and Suppliers (COL), and Implementation of Dedicated Logistics Department (DLD) are employed in the design of the fuzzy logic system. Fig, 4 illustrates the conceptual architecture of the fuzzy logic system used in the framework design. 


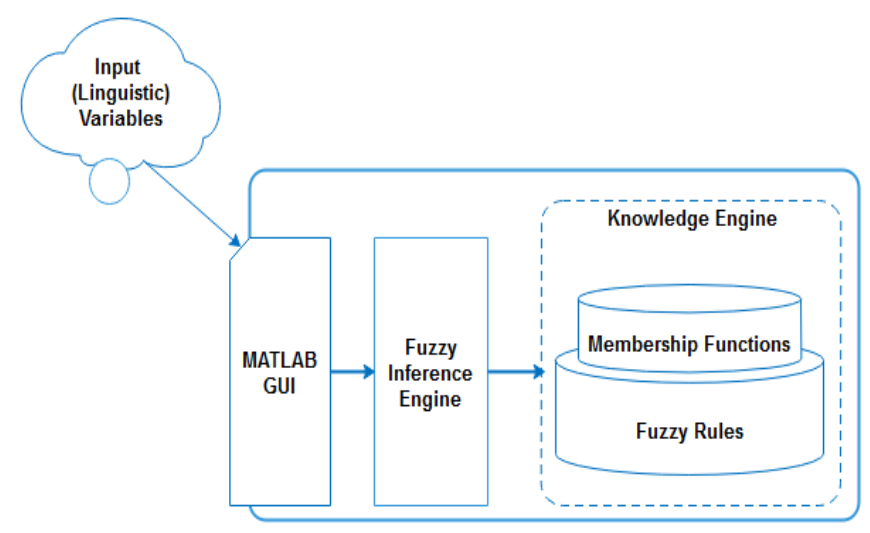

Fig. 4. Conceptual Framework for Architecture of the FIS.

\section{F. Fuzzification}

We performed a fuzzification simulation on the values of the selected input variables using the membership functions to determine their degree of membership. This converts the crisp quantities into fuzzy values. This is then used to map the output value specified in the individual rules to an intermediate output measuring fuzzy sets. The fuzzy linguistics variables and terms for each input parameter are defined as follows;

1) Logistics Optimization [Poor, Good, and Excellent].

2) Enabling Information/Cognitive Technology [None, Minimal, and Maximal].

3) Strategic Collaboration of all stakeholders and suppliers [Low, Medium, and High].

4) Implementation of Dedicated Logistic Department [None, Undefined, Adopted].

The output fuzzy linguistic variable and its terms are defined as Supply Chain Efficiency [Inefficient, Average, and Efficient]. The universe of discourse for the input and output parameters are all defined using a scale of impact significance thus;

1) Logistics Optimization $[0,10]$. $10]$.

2) Information/Cognitive Technology Adaptability [0,

3) Strategic Collaboration of Stakeholders and Suppliers $[0,10]$.

4) Implementation of a Dedicated Logistics Department $[0,10]$.

5) Supply Chain Efficiency [0, 10], respectively.

The crisp input and output values are converted to fuzzy values by the input and output Membership Functions (MFs) respectively. A Triangular membership functions (MFs) is used for the evaluation. A Triangular MF curve depends on three parameters b1; b2, and b3, as illustrated below;

$\mu(x)= \begin{cases}0(x-b 1) /(b 2-b 1) & \text { if } x<1 \leq x<b 2 \\ 0(b 3-x) /(b 3-b 2) & \text { if } b 2 \leq x<b 3 \\ \text { if } x>b 3,\end{cases}$

where b2 defines the triangular peak location, while b1 and b3 defines the triangular end points.

\section{G. Membership Function Definition}

In defining our membership functions, we have employed the triangular membership function in our fuzzy inference system. Individual range of inputs and output variables are outlined to relate with a fuzzy set that has the same name as the range. We have identified four Linguistic Input Variables and defined three fuzzy sets for these input variables as well as three fuzzy sets for the output variable. The Triangular Membership Functions are as defined the equations.

$L O(x)=\left\{\begin{array}{c}\text { if } 0 \leq x \leq 4, \mid " \text { Poor" } \\ \text { if } 3 \leq x \leq 7, \mid " G o o d " \\ \text { if } 6 \leq x \leq 10, \mid " \text { Excellent" }\end{array}\right.$

$\operatorname{ETA}(x)=\left\{\begin{array}{c}\text { if } 0 \leq x \leq 4, \mid " N o n e " \\ \text { if } 3 \leq x \leq 7, \mid " M i n i m a l " \\ \text { if } 6 \leq x \leq 10, \mid " M a x i m a l "\end{array}\right.$

$\operatorname{CoL}(x)=\left\{\begin{array}{l}\text { if } 0 \leq x \leq 4, \mid " L o w " \\ \text { if } 3 \leq x \leq 7, \mid \text { Medium" } \\ \text { if } 6 \leq x \leq 10, \mid " H i g h "\end{array}\right.$

$D L D(x)=\left\{\begin{array}{c}\text { if } 0 \leq x \leq 4, \mid " N o n e " \\ \text { if } 3 \leq x \leq 7, \mid " \text { Undefined" } \\ \text { if } 6 \leq x \leq 10, \mid " \text { Adopted" }\end{array}\right.$

$\operatorname{SCE}(x)=\left\{\begin{array}{l}\text { if } 0 \leq x \leq 4, \mid " \text { Inefficient" } \\ \text { if } 3 \leq x \leq 7, \mid " \text { Average" } \\ \text { if } 6 \leq x \leq 10, \mid " \text { Efficient" }\end{array}\right.$

The fuzzy rules are defined based on observations and analysis from our structure literature review and data collected from the case studies. Fig. 5 shows membership plots shows how the rules are applied in constructing the different membership plots.

\section{H. Fuzzy System Evaluation}

The subsequent simulation results from our fuzzy evaluation were obtained as we varied the weight factors (input variables) of the control coefficients which we had initially defined on a scale where our weight factors lied between one and ten. Fig. 6 to 7 presents sections of our simulation with different input parameters.

From Fig. 5(a), the input variables, LO is set at 5 which portrays Good, ETA is set at 5 which portrays Minimal, COL is set at 5 which portrays Medium and DLD is set at 5 which portrays Undefined and just as the rules define, our output: Supply Chain Efficiency is set at 5 and according to our defined rules means the Supply Chain efficiency is average. By the output being average, the system indicates that if in performing a Healthcare Logistics Evaluation, the Logistics Optimization (LO) is GOOD, the use of Information/Cognitive Technology (ETA) is MINIMAL, there is a MEDIUM Collaboration of all Stakeholders and Suppliers (COL) and the Healthcare facility's is just basic or UNDEFINED then the Supply Chain Efficiency (SCE) will be AVERAGE. 


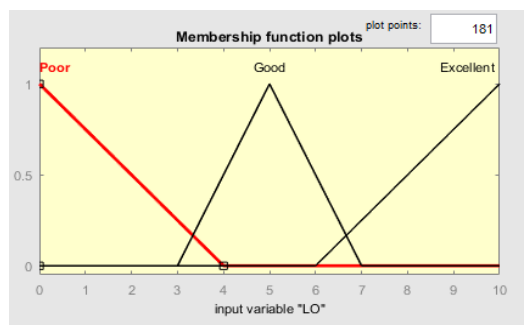

(a): Logistic Optimization (LO).

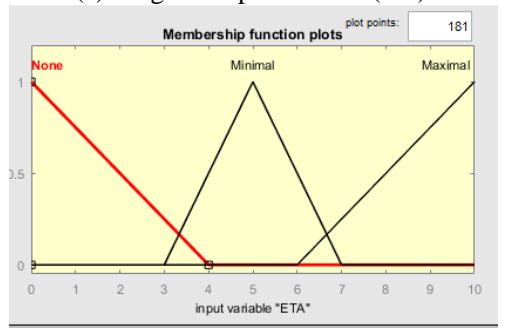

(b): Information/Cognitive Technology Adaptation (ETA).

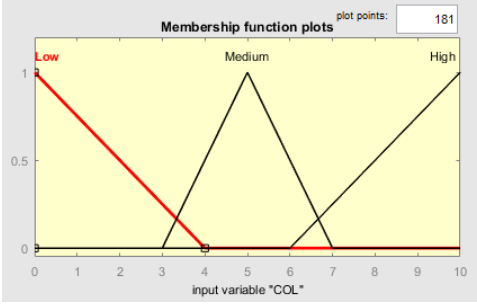

(c): Collaboration of all Stakeholders and Suppliers (COL).



(d): Implementation of Dedicated Logistic Department (DLD).

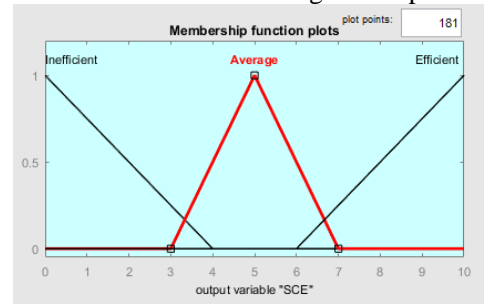

Fig. 5. (e): Supply Chain Efficiency (SCE).

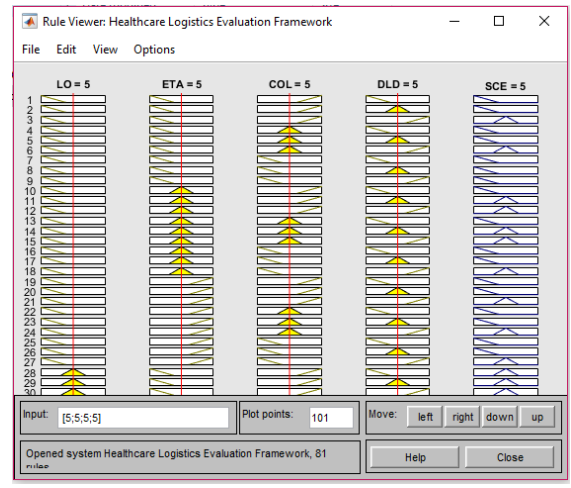

Fig. 6. First Simulation Instance.

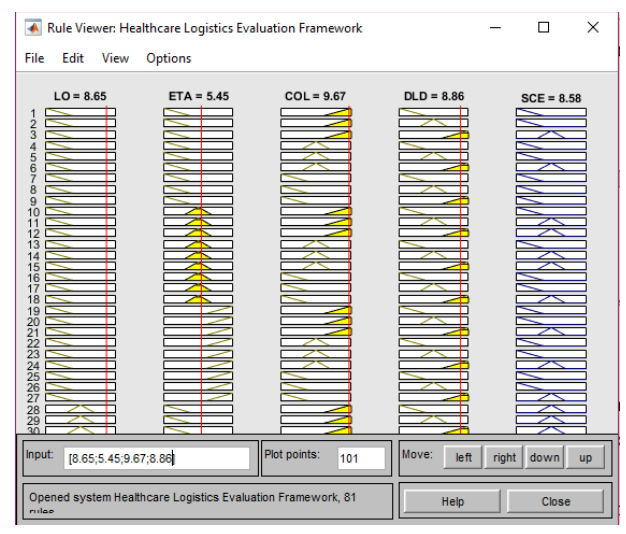

Fig. 7. Second Simulation Instance.

From our second simulation instance, the input variables, LO is set at 8.65 which portrays EXCELLENT Logistics Optimization, ETA is set at 5.45 which portrays MINIMAL usage of IT, COL is set at 9.67 which portrays a very HIGH collaboration with all stakeholders and suppliers and DLD is set at 8.86 which indicates that the hospital has ADOPTED or has a Dedicated Logistics Department and based on these inputs the system predicts that the Supply Chain Efficiency (SCE) is 8.85 which indicates that Supply Chain if implemented will be very EFFICIENT.

The surface viewer allows us to see the entire output surface of our inference system - the entire span of the output set based on the entire span of the input set. Below we present the surface plots of the input variables that impact our Logistics Evaluation Framework.

Fig. 8 presents a Surface Plot for LO (Logistic Optimization) against DLD (Implementation of a Dedicated Logistic Department). This surface plot shows that the Supply Chain is moves towards high efficiency when Logistics Optimization is EXCELLENT and the healthcare facility has a Dedicated Logistics Department to manage the Logistics affairs thus reducing the bottleneck that often arises from health workers having to combine performing logistics activities and their primary responsibilities.

Similarly in Fig. 9, COL (Collaboration of all Stakeholders and Suppliers) is plotted against ETA (Adaptation of Information/Cognitive Technologies), and from this we see that both parameters need to be at their highest (i.e. High and Maximal respectively) for the efficiency of the supply chain to move towards attaining efficiency.

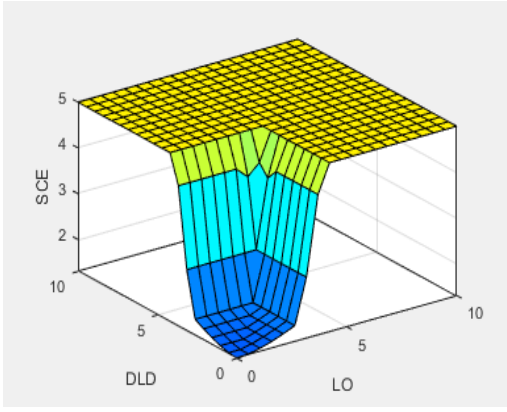

Fig. 8. Surface Plot for LO Against DLD. 


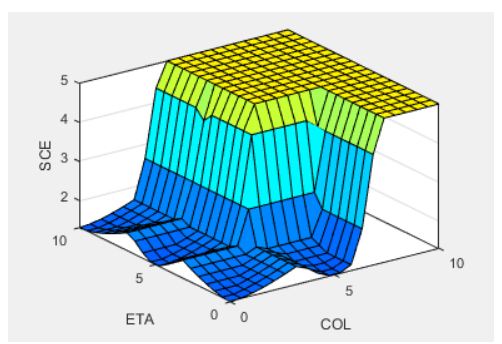

Fig. 9. Surface Plot for COL against ETA.

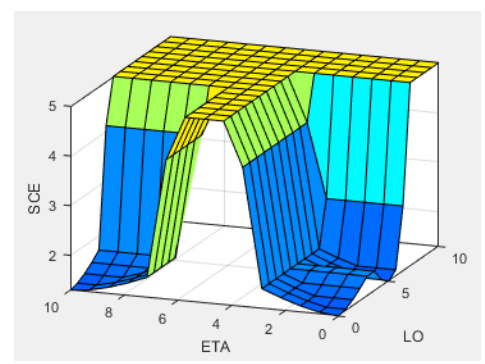

Fig. 10. Surface Plot for LO against ETA.

In Fig. 10, we consider how ETA (Adaptation of Information/Cognitive Technologies) is represented against LO (Logistics Optimization) and from the above it shows that optimizing logistics and adapting information and cognitive technologies to healthcare supply chain process will contribute to over $50 \%$ percent of the efficiency of the Supply Chain.

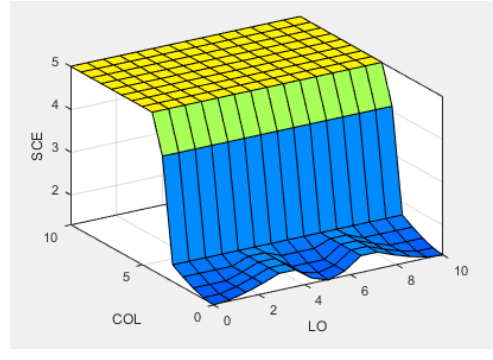

Fig. 11. Surface Plot for $\mathrm{LO}$ against COL.

Fig. 10 presents the impact metrics on Supply Chain Efficiency (SCE) when Logistics Processes are optimized (LO) and there is a collaboration of all Logistics stakeholders (COL). From the Fig. 11 we see that Logistics optimization has a great impact on the efficiency of the supply chain. It shows that when Hospital logistics are optimized, the more emphasis would be laid on collaborating logistics and medical stakeholders. Although this collaboration is necessary, the effect of omitting it is minimal and cannot be termed as fatal to the efficiency of our intended supply chain.

Similarly, Fig. 12 shows how the collaboration of stakeholders (COL) performs when compared against Adaptation of Information/Cognitive Technologies (ETA). Though the collaboration of Stakeholders (COL) affects the efficiency curve of the supply chain in this case, ETA affects the efficiency of the Supply Chain more than collaborating stakeholders further highlighting the importance of incorporating advancement in information technologies (IT) and cognitive science to the medical logistics and supply chain framework.

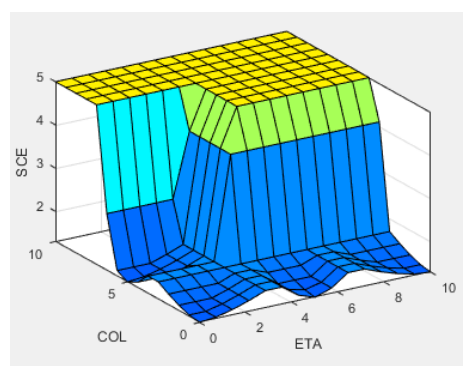

Fig. 12. Surface Plot for COL against ETA.

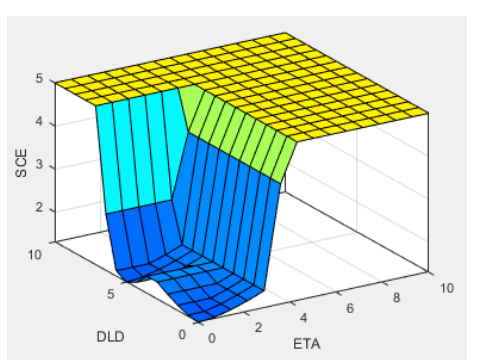

Fig. 13. Surface Plot for DLD against ETA.

Fig. 13 compares two of the variables with the very high coefficient constraints: incorporating a Dedicated Logistics Department (DLD) and Adaptation of Information and Cognitive Technologies (ETA). Both contributes similarly to the efficiency of the supply chain. These two variables are the backbone of the logistics optimization framework for efficient supply chain.

\section{Evaluation Framework PRESENTATION}

The Control Coefficients presented in Table IV presents the Decision Criteria for the design of our evaluation framework. The framework is strategically important in analysis, evaluation and optimization of Healthcare Logistics activities, processes and operations and considering that Healthcare Logistics is an integral and inseparable component of Healthcare Supply Chain therefore any improvement achieved by our Healthcare Logistics Evaluation and Optimization Framework subsequently improves the efficiency of the Healthcare Supply Chain implementation. The framework extrapolates the relationship that exists among the control coefficients as well as their bearing on the Healthcare Logistics Activities and Processes. Our framework presents a strategy for managing Healthcare Logistics Operations for the purpose of ensuring optimization and efficiency of Logistics Activities within any Healthcare Institution while effecting the realization of an Efficient Supply Chain in the Healthcare Sector.

The Healthcare Logistics Evaluation Framework presented in Fig. 14 examines and explicates the bottleneck experienced by the practice of shared management Logistics Activities by Designated Logisticians - in very few cases and Medical Personnel-Doctors and nurses, who by this shared responsibility only devote half their time and effort in performing their primary responsibilities. 


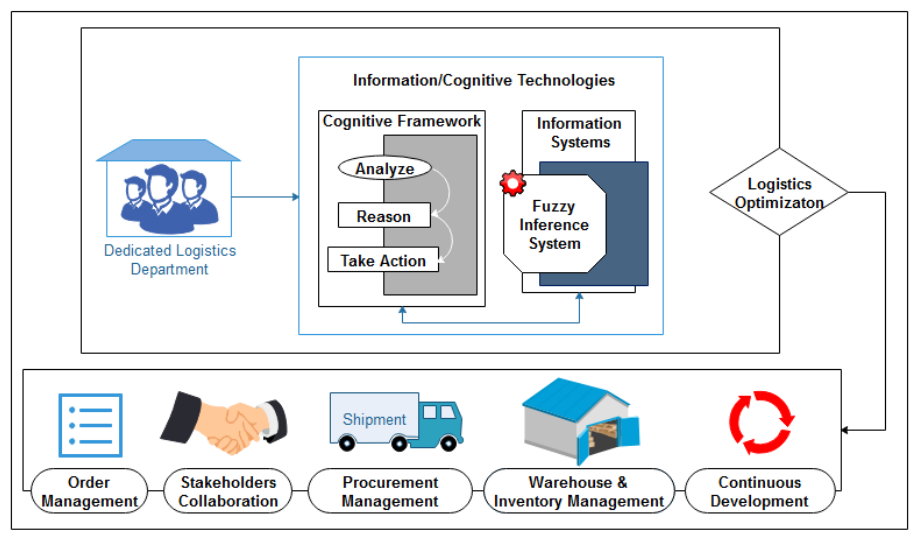

Fig. 14. Proposed Healthcare Logistics Evaluation Framework.

\section{CONCLUSION}

With increasing demands on healthcare providers and hospital managers in providing Quality of Care (QoC), better Hospital resource management and allocation as well as Quality Healthcare service delivery. Indeed, the search for better approaches of managing hospital processes has increased exponentially. Given today's reality when information technologies and Intelligence Systems are the order of the day, Healthcare managers must catch up and apply cognitive approaches and top-notch techniques in information technology to tackle the problems in the healthcare industry. A very great way to begin this adoption is to better manage the thing affecting every stakeholder in the Healthcare Industry-Logistics. Considering this narrative, our framework is a timely solution to healthcare logistics evaluation and optimization for efficient supply chain.

\section{ACKNOWLEDGMENT}

Special Acknowledgement to TETFUND, Nigeria for Supporting Research and Development (R\&D) in Tertiary Institutions in Nigeria.

\section{REFERENCES}

[1] Al-Qatawneh, L., Abdallah, A. A. \& Zalloum S. Z. (2019). Six sigma application in Heathcare Logistics: A framework and a case study. Journal of Health Engineering 2019, ID: 9691568 DOI: https://doi.org/10.1155/2019/9691568.

[2] Campbell M., Fitzpatrick R., Haines A., Kinmonth A. L., Sandercock P., Spiegelhalter D., \& Tyrer P. (2000). Framework for design and evaluation of complex interventions to improve health. BMJ, Volume 321.

[3] Carlos F. \& Alfonso-Lizarazo E. (2017). A structured review of quantitative models of the pharmaceutical supply chain; Universidad del Rosario, Escuela de Administraci'on, Bogot'a, Colombia.

[4] Chen, H. K., Chen, H. Y., Wu, H. H., \& Lin, W. T. (2004). TQM Implementation in a Healthcare and Pharmaceutical Logistics Organization: The Case of Zuellig Pharma in Taiwan. Total Quality Management \& Business Excellence, 15(9-10), 1171-1178.

[5] Denton B. T. (Ed.). (2013). Handbook of Healthcare Operations Management: Methods and Applications. International Series in Operations Research Management Science 184, DOI 10.1007/978-14614-5885-2_3. Springer Science+Business Media New York.

[6] Dubey R., Gunasekaran A., Papadopoulos T., Childe S. J., Shibin K. T., Wamba, S. F. (2016) Sustainable supply chain management: framework and further research directions. Journal of Cleaner Production, 142(2): 1119-1130. ISSN 09596526.
[7] Feibert, D. C. (2017). Improving healthcare logistics processes. DTU Management Engineering, Technical University of Denmark: PhD Thesis. Retrieved from orbit.dtu.dk on January 26, 2020.

[8] Feibert, D. C. \& Jacobsen, P. (2015). Measuring process performance within healthcare logistics - a decision tool for selecting track and trace technologies. Academy of Strategic Management Journal, 14. Special issue, 33-57.

[9] Frichi Y., Jawab f., Boutahari S., Zehmed K., Moufad I., Akoudad K. \& Laaraj N. (2018): Hospital Logistics; an effective tool in improving the quality of care; Laboratory of manufacturing, energy and sustainable development; Sidi Mohamed Ben Abdellah University Fez, Morocco.

[10] Gang D., Xi Liang, \& Chuanwang S. (2017). Scheduling Optimization of Home Health Care Service Considering Patients' Priorities and Time Windows. Department of Business Management, School of Business Administration, East China Normal University, Shanghai 200062, China/.

[11] Hugos M. (2003). Essentials of supply chain management. John Wiley \& Sons, Inc., Hoboken, New Jersey.

[12] Ioannis Nikolaou, Konstantinos I. Evangelinos and Stuart Allan (2013). A reverse logistics social responsibility evaluation framework based on the triple bottom line approach, Journal of Cleaner Production 56:173$184 /$.

[13] Jawab F., Frichi Y. \& Boutahari S. (2018). Hospital Logistics Activities. Proceedings of the International Conference on Industrial Engineering and Operations Management Bandung, Indonesia, 3228-3237/.

[14] Kazemzadeh R. B., Sepehri M. M. \& Jahantigh F. F. (2012). The drug logistics process: an innovative experience, The TQM Journal Vol. 27 No. 2, 2015pp. 214-230@ Emerald Group Publishing Limited 17542731 DOI 10.1108/TQM-01-2015-0004/.

[15] Kumar, A. \& Rahman, S. (2014). RFID-Enabled Process Reengineering of Closed loop Supply Chains in the Healthcare Industry of Singapore. Journal of Cleaner Production, Elsevier Ltd, 85, 382-394.

[16] Kriegel J., Jehle F., Dieck M. \& Mallory P. (2013). Advanced services in hospital logistics in the German health service sector. Logistics Research, 6(2-3), 47-56.

[17] Kriegel J., Jehle F., Dieck, M. \& Tuttle-weidinger L. (2015). Optimizing patient flow in Austrian hospitals-Improvement of patient-centered care by coordinating hospital-wide patient trails. International Journal of Healthcare Management, 8(2), 89-99.

[18] Manso J. F., Annan J. \& Anane S. S. (2017). Assessment of Logistics Management in Ghana Health Service. International Journal of Business and Social Research 3(8), 75-87.

[19] Memon, Z., Noran, O. \& Bernus, P. (2019). A framework to evaluate architectural solutions for ubiquitous patient identification in health information systems. Proceedings of the 21st International Conference on Enterprise Information Systems, 580-587. ISBN: 978-989-758-372-8.

[20] Najafi M., Eshghi K., \& Dullaert, W. (2013). A multi-objective robust optimization model for logistics planning in the earthquake response phase. Transportation Research Part E, 49(1), 217-249. 
[21] Ohagim I. P., Nyong E. E. \& Moses A. E. (2018). Methicillin-resitant staphylococcus aureus nasal carriage among surgical patients, patient relatives and healthcare workers in a teaching hospital in Uyo, Southsouth Nigeria. Journal of Advances in Microbiology 8(1): 1-11.

[22] Pinna R., Carrus P. P. \& Marras F. (2015). The drug logistics process: an innovative experience. The TQM Journal, 27(2), 214-230.

[23] Pohjosenperä, T., Kekkonen, P., Pekkarinen, S., \& Juga, J. (2019). Service modularity in managing healthcare logistics. The International Journal of Logistics Management, 30(1), 74-194.

[24] Umego C. F., Mboto C. I., Mbim E. N., Edet U. O., George U. E. \& Tarh J. E. (2018). Epidemiology of hepatitis B virus infection in SouthSouth, Nigeria: a review. International STD Research \& Reviews 7(1): $1-17$.
[25] Umoren I., Udonyah K. \& Isong E. (2019). Computational Intelligence Framework for Length of Stay Prediction in Emergency Healthcare Services Department. IEEE Xplore Digital Library, 2473-9464. DOI: 10.1109/ICCSE.2019.8845332.

[26] Umoren I., Usua G. and Osang F. (2019). Analytic Medical Process for Ophthalmic Pathologies Using Fuzzy C-Mean Algorithm, Article Innovations in Systems and Software Engineering.

[27] Wang G., Gunasekaran A., Ngai E. W. T. \& Papadopoulos, T. (2016). Big data analytics in logistics and supply chain management: Certain investigations for research and applications. International Journal of Production Economics, 176: 98-110. ISSN 09255273. 